\title{
A simple and non-conventional method for the synthesis of selected $\beta$-arylalkylchalcogeno substituted alcohols, amines and carboxylic acids
}

\author{
Patrícia C. Silva, ${ }^{a}$ EIton L. Borges, ${ }^{\text {b }}$ David B. Lima, ${ }^{\mathrm{b}}$ Raquel G. Jacob, ${ }^{\mathrm{b}}$ Eder \\ J. Lenardão, ${ }^{\mathrm{b}}$ Gelson Perin, ${ }^{\mathrm{b}, *}$ Márcio S. Silva ${ }^{\mathrm{a}, *}$ \\ ${ }^{a}$ Centro de Ciências Naturais e Humanas (CCNH), Universidade Federal do ABC, \\ Av. dos Estados 5001, 09210-580, Santo André, SP, Brazil. \\ ${ }^{b}$ LASOL - CCQFA - Universidade Federal de Pelotas - UFPel - P.O. Box 354 - 96010-900, \\ Pelotas, RS, Brazil. \\ E-mail:s.marcio@ufabc.edu.br; gelson_perin@ufpel.edu.br
}

DOI: $\underline{\text { https://doi.org/10.24820/ark.5550190.p009.906 }}$

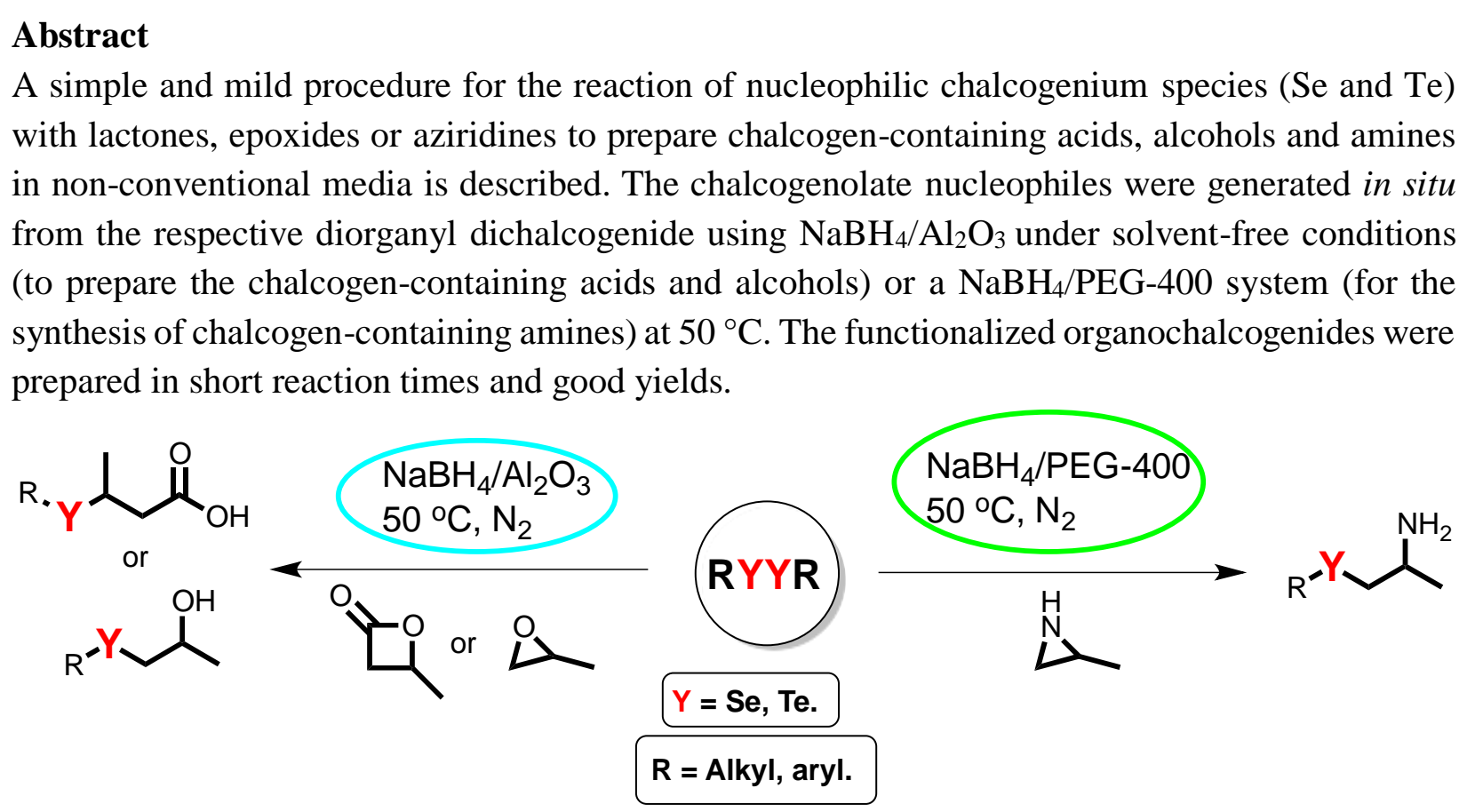

Keywords: organochalcogen compounds; lactone; epoxide; aziridine; ring opening reaction.

\section{Introduction}

The development and applications of organoselenium and organotellurium compounds are well known. ${ }^{1-3}$ Organochalcogen compounds, when associated with other functionalities, are versatile 
reagents in organic synthesis. ${ }^{4}$ Additionally, recently they have been applied in materials science, ${ }^{5}$ biological and pharmacological studies ${ }^{6}$ and organic synthesis. In view of these features, the search for greener reaction conditions and efficient methodologies to reduce wastes in the synthesis of new organochalcogen compounds has received much attention. ${ }^{7-11}$

On the other hand, the ring opening reaction by nucleophilic selenium and tellurium species is a very useful method to incorporate these elements into organic molecules, due to their soft nucleophilicity and low basicity. The use of cleaner procedures for producing nucleophilic species of organochalcogen has been an efficient strategy. Among the alternative methods employed for generation in situ of chalcogenolate anions for ring opening reactions of epoxides, aziridines and lactones are: $\mathrm{Zn} / \mathrm{HCl} /$ biphasic, ${ }^{12} \quad \mathrm{Zn} / \mathrm{THF} / \mathrm{reflux},{ }^{13} \quad \mathrm{Zn} / \mathrm{HCl} /[\mathrm{bmim}]\left[\mathrm{BF}_{4}\right],{ }^{14}$ $\mathrm{KOH} / \mathrm{CuO} /[\mathrm{bmim}]\left[\mathrm{BF}_{4}\right]^{15}$ and $\mathrm{Zn} / \mathrm{AlCl}_{3} / \mathrm{CH}_{3} \mathrm{CN} / 70{ }^{\circ} \mathrm{C} .{ }^{16}$ In most cases, these methods employ strong base or acid, high temperatures and volatile organic compounds (VOCs), limiting their use to a few functional groups.

Recently, we developed a new method to the in situ generation of chalcogenolate anions by using the $(\mathrm{RY})_{2} / \mathrm{NaBH}_{4} / \mathrm{PEG}-400$ system. This protocol was successfully used to prepare vinyl chalcogenides, ${ }^{17}$ bis-chalcogen alkenes ${ }^{18}$ and $\beta$-chalcogen esters, ketones and carboxylic acids. ${ }^{19,20}$ By this procedure, the use of odoriferoous, unstable compounds and drastic reaction conditions are avoided, enabling us to explore the soft nucleophilicity of organochalcogen ( $\mathrm{Se}$ and $\mathrm{Te}$ ) compounds under mild conditions.

In this work, we describe the ring-opening reaction of lactones, epoxides and non-activated aziridines by chalcogenolate anions using solvent-free $\mathrm{NaBH}_{4} / \mathrm{Al}_{2} \mathrm{O}_{3}$ or $\mathrm{NaBH} / \mathrm{PEG}-400$ systems at $50{ }^{\circ} \mathrm{C}$ to prepare chalcogen-functionalized carboxylic acids, alcohols and amines (Scheme 1).

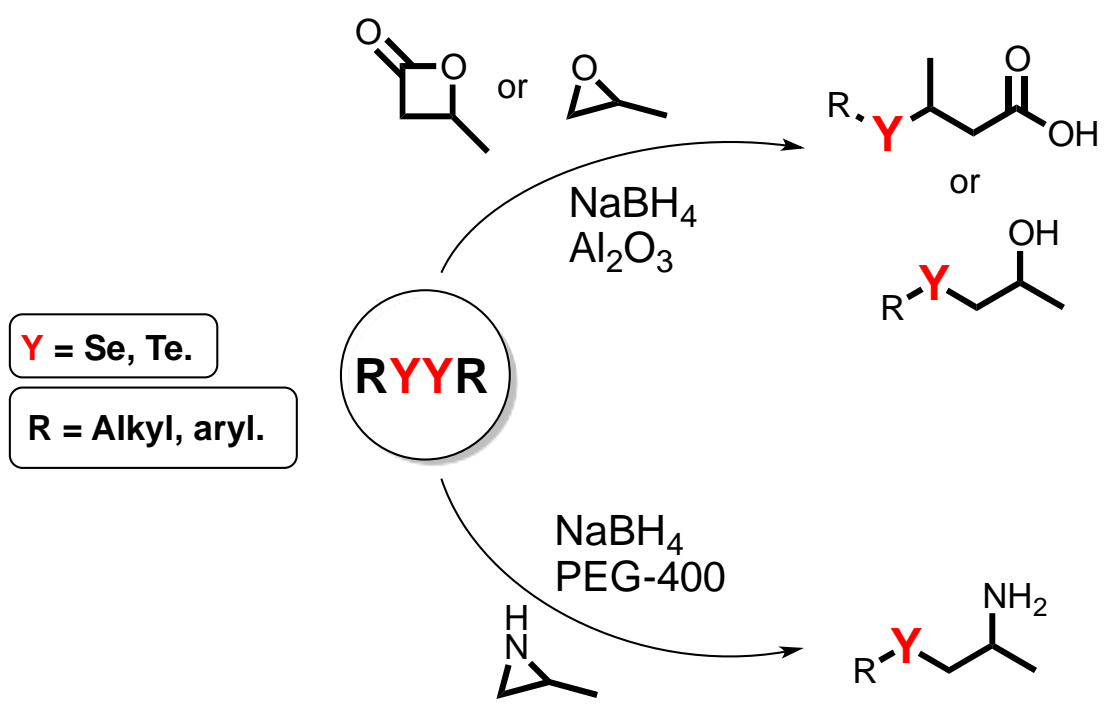

Scheme 1. Synthesis of chalcogen-containing acids, alcohols and amines under mild conditions. 


\section{Discussion}

In the initial experiments, we chose diphenyl ditelluride 1a as the chalcogen source and $\beta$-methyl$\beta$-propiolactone 2a, propylene oxide $\mathbf{2 b}$ and 2-methylaziridine $\mathbf{2 c}$ as the electrophiles to evaluate the best conditions for the nucleophilic ring-opening reaction (Table 1). In the optimization study, we examined the influence of the amount of electrophile, the temperature and the use of solvent or solid-supported reducing agent. It was observed that the presence of solid support or solvent is essential for the success of the ring-opening of the three electrophiles (Table 1, entries 1, 17 and 22). As can be seen in Table 1, the use of $50.0 \mathrm{mg}$ of solid support or $50 \mu \mathrm{L}$ of solvent and an excess of electrophile $(1.5 \mathrm{mmol})$ provided the best results (entries 5, 13 and 20). The reactions were monitored by thin layer chromatography (TLC) and gas chromatography (GC).

Among the conditions that were tested for the ring-opening of lactone 2a to obtain the tellurium-containing acid 3a, the most effective approach was that using $\mathrm{NaBH}_{4} / \mathrm{Al}_{2} \mathrm{O}_{3}$ at $50{ }^{\circ} \mathrm{C}$, which afforded the product in $76 \%$ yield (Table 1, entry 3 ). A decrease in the yield of 3a was observed when the reaction was performed either at room temperature or at $80^{\circ} \mathrm{C}$ (Table 1, entries 2 and 4), while $83 \%$ of the product was obtained using an excess of $2 \mathbf{a}$ ( 1.5 equiv) with respect to the ditelluride 1a (Table 1, entry 5). We also tested alternative solvents in the reaction, such as PEG-400, glycerol and ethanol. A satisfactory yield of $\mathbf{3 a}$ was obtained only when $\mathrm{NaBH}_{4} / \mathrm{PEG}-$ 400 was used (Table 1, entries 9-10). Ethanol delivered 3a in only 34\% yield, while using glycerol caused the formation of a solid in the reaction vessel, thus preventing mixing of reagents (Table 1, entries 11-12). When propylene oxide $\mathbf{2} \mathbf{b}$ was used as the electrophile, the profile of the reaction remained the same, with the $\mathrm{NaBH}_{4} / \mathrm{Al}_{2} \mathrm{O}_{3}$ system, affording the desired tellurium-containing alcohol $4 \mathrm{a}$ in $88 \%$ yield after $2 \mathrm{~h}$ at $50{ }^{\circ} \mathrm{C}$ (Table 1, entry 13). The $\mathrm{NaBH}_{4} / \mathrm{PEG}-400$ system afforded the alcohol $4 \mathbf{a}$ in $80 \%$ yield (Table 1, entry 15). We observed that $\mathrm{SiO}_{2}$ is not as good as $\mathrm{Al}_{2} \mathrm{O}_{3}$ as the solid support and the ring-opening was less efficient for both lactone $\mathbf{2 a}$ and epoxide $\mathbf{2 b}$ (Table 1, entries 8 and 14). Ethanol was not a good solvent to prepare $\mathbf{4 a}$, which was obtained in only $41 \%$ yield after $2 \mathrm{~h}$ (Table 1, entry 16). A longer reaction time did not improve the yield.

In striking contrast to these results, $\mathrm{NaBH}_{4} / \mathrm{PEG}-400$ system gave the best result in the ringopening of 2-methylaziridine $\mathbf{2 c}$, affording the tellurium-containing amine $\mathbf{5 a}$ in $76 \%$ yield (Table 1, entry 20). Clearly, to produce chalcogen amines the presence of a hydrogen source is essential for success. These results are corroborated by those using conventional methodologies. ${ }^{21,22}$ However, when the reaction was carried out in ethanol, the yield of $\mathbf{5 a}$ decreased to $57 \%$ (Table 1, entry 21). The use of additional ethanol $(2.0,5.0$ and $10.0 \mathrm{~mL})$ did not change the outcome. Neither increasing the temperature $\left(80\right.$ and $\left.100{ }^{\circ} \mathrm{C}\right)$ nor using a larger excess of aziridine $2 \mathbf{c}(2.0,3.0$ and 5.0 equiv) improved the yield of $\mathbf{5 a}$ using the $\mathrm{NaBH}_{4} / \mathrm{PEG}-400$ system. 
Table 1. Optimization of the ring-opening reactions of lactone, epoxide and aziridine by phenyltellurolate anion $^{\mathrm{a}}$

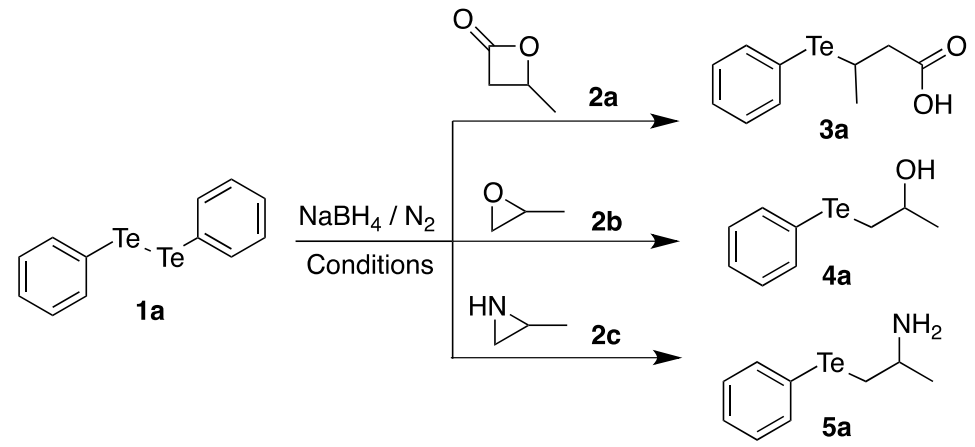

\begin{tabular}{|c|c|c|c|c|}
\hline Entry & $\begin{array}{l}\text { Conditions } \\
\text { (mg or } \square \mathrm{L} \text { ) }\end{array}$ & $\begin{array}{l}\text { Electrophile } \\
\text { (mmol) }\end{array}$ & Temp. $\left({ }^{\circ} \mathrm{C}\right)$ & Yield $(\%)^{b}$ \\
\hline 1 & --- & $\stackrel{2 a}{2 a}_{(1.0)}^{o+o}$ & 25 & 22 \\
\hline 2 & $\mathrm{Al}_{2} \mathrm{O}_{3}(50)$ & $\mathbf{2 a}(1.0)$ & 25 & 53 \\
\hline 3 & $\mathrm{Al}_{2} \mathrm{O}_{3}(50)$ & $\mathbf{2 a}(1.0)$ & 50 & 76 \\
\hline 4 & $\mathrm{Al}_{2} \mathrm{O}_{3}(50)$ & $\mathbf{2 a}(1.0)$ & 80 & 55 \\
\hline 5 & $\mathrm{Al}_{2} \mathrm{O}_{3}(50)$ & $2 \mathrm{a}(1.5)$ & 50 & 83 \\
\hline 6 & $\mathrm{Al}_{2} \mathrm{O}_{3}(100)$ & $\mathbf{2 a}(1.5)$ & 50 & 73 \\
\hline 7 & $\mathrm{Al}_{2} \mathrm{O}_{3}(50)$ & $\mathbf{2 a}(2.0)$ & 50 & 80 \\
\hline 8 & $\mathrm{SiO}_{2}(50)$ & $\mathbf{2 a}(1.5)$ & 50 & 63 \\
\hline 9 & PEG-400 (50) & $\mathbf{2 a}(1.5)$ & 50 & 78 \\
\hline 10 & PEG-400 (100) & $\mathbf{2 a}(1.5)$ & 50 & 67 \\
\hline 11 & ethanol (50) & $\mathbf{2 a}(1.5)$ & 50 & 34 \\
\hline 12 & glycerol (50) & $\mathbf{2 a}(1.5)$ & 50 & ---- \\
\hline 13 & $\mathrm{Al}_{2} \mathrm{O}_{3}(50)$ & $\stackrel{Q}{2 b}_{(1.5)}$ & 50 & 88 \\
\hline 14 & $\mathrm{SiO}_{2}(50)$ & $\mathbf{2 b}(1.5)$ & 50 & 71 \\
\hline 15 & PEG-400 (50) & $\mathbf{2 b}(1.5)$ & 50 & 80 \\
\hline 16 & ethanol (50) & $2 \mathbf{b}(1.5)$ & 50 & 41 \\
\hline 17 & ---- & $2 \mathbf{b}(1.5)$ & 50 & 32 \\
\hline 18 & $\mathrm{Al}_{2} \mathrm{O}_{3}(50)$ & $\sum_{2 \mathrm{c}}^{\stackrel{\mathrm{H}}{\mathrm{N}}}(1.5)$ & 50 & 48 \\
\hline 19 & $\mathrm{SiO}_{2}(50)$ & $2 \mathrm{c}(1.5)$ & 50 & 25 \\
\hline 20 & PEG-400 (50) & $2 c(1.5)$ & 50 & 76 \\
\hline 21 & ethanol (50) & $2 \mathrm{c}(1.5)$ & 50 & 57 \\
\hline 22 & ---- & $2 \mathrm{c}(1.5)$ & 50 & 18 \\
\hline
\end{tabular}

${ }^{a}$ Reaction performed in the presence of $0.5 \mathrm{mmol}$ of diphenyl ditelluride $1 \mathrm{a}$ and $1 \mathrm{mmol}^{\text {of } \mathrm{NaBH}_{4}}$ for $2 \mathrm{~h} .{ }^{\mathrm{b}}$ Yields are given for isolated products $\mathbf{3 a}, \mathbf{4 a}$ or $\mathbf{5 a}$. 
With the standard reaction conditions defined, we next investigated the scope of our methodology by employing a variety of chalcogenolate anions and electrophiles. To establish the generality for the lactone $\mathbf{2 a}$ ring-opening reaction, various diorganyl dichalcogenides $\mathbf{1}$ were used in the presence of $\mathrm{NaBH}_{4} / \mathrm{Al}_{2} \mathrm{O}_{3}$ system at $50{ }^{\circ} \mathrm{C}$. The reactions proceeded with good yields employing diaryl and dialkyl dichalcogenides and they are not sensitive to electronic effects in the aromatic ring of the diaryl ditellurides (Scheme 2). This approach was successfully extended to diphenyl diselenide 1e and the respective $\beta$-phenylselanyl carboxylic acid $\mathbf{3 e}$ was obtained in $85 \%$ yield (Scheme 2). A good result was obtained when $\gamma$-butyrolactone $\mathbf{2 d}$ was reacted with diphenyl diselenide 1e in the presence of $\mathrm{NaBH}_{4} / \mathrm{Al}_{2} \mathrm{O}_{3}$, yielding the $\gamma$-phenylselanyl acid $\mathbf{3 f}$ in $79 \%$ yield (Scheme 3).
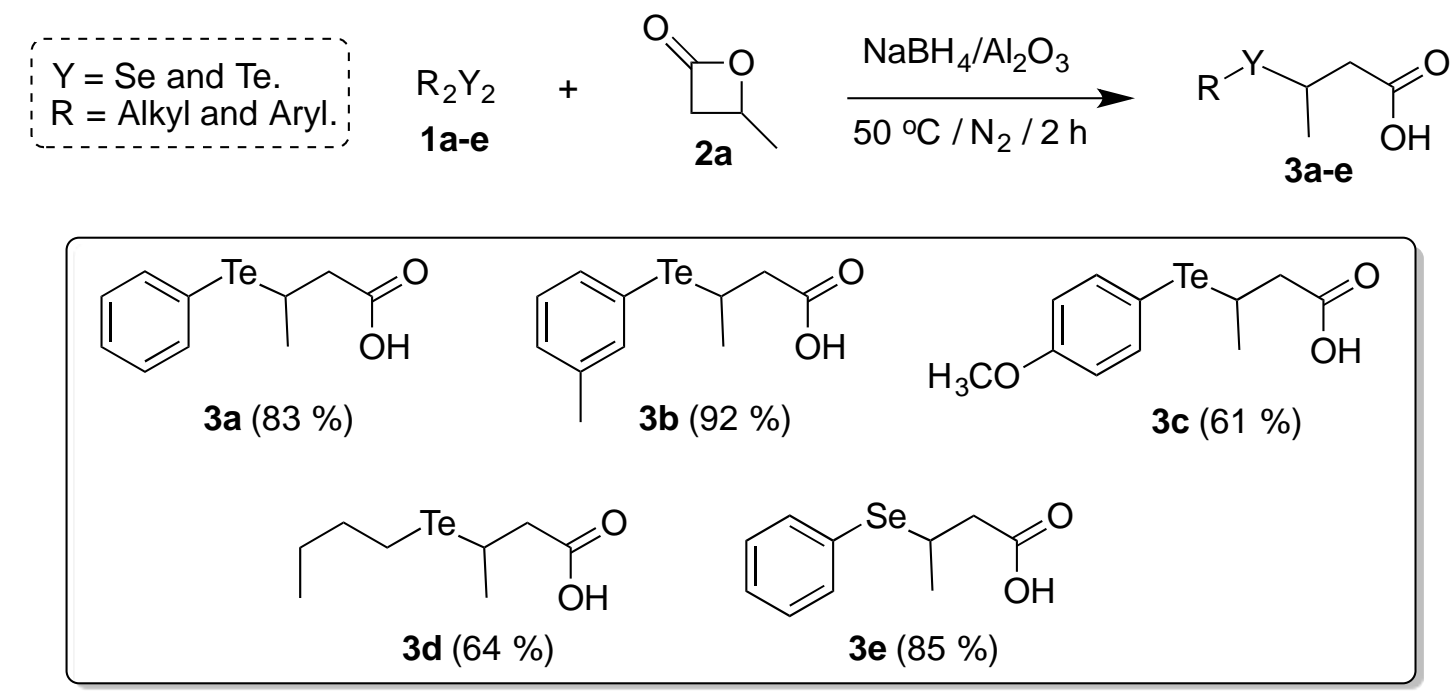

Scheme 2. Synthesis of $\beta$-organylchalcogenyl acids 3a-e by the ring-opening reaction of $\beta$ methyl- $\beta$-propiolactone $\mathbf{2 a}$.

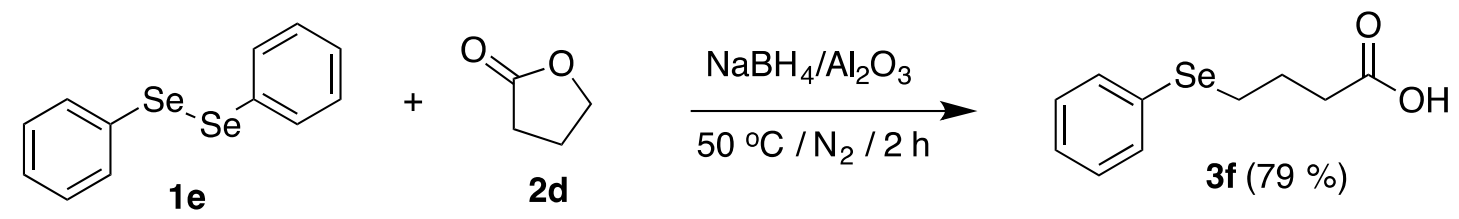

Scheme 3. Synthesis of the $\gamma$-phenylselanyl acid $\mathbf{3 f}$ by the ring opening reaction of $\gamma$-butyrolactone 2d.

Excellent results were obtained in the reaction of the chalcogenolate anions generated in situ with epoxides (Scheme 4). As can be seen in Scheme 4, $\beta$-chalcogen alcohols 4a-k were obtained in good to excellent yields from different epoxides and a variety of ditellurides and diselenides. For instance, 2-benzyloxirane 2e reacted with diphenyl ditelluride 1a and diphenyl diselenide 1e in the presence of $\mathrm{NaBH}_{4} / \mathrm{Al}_{2} \mathrm{O}_{3}$ to afford, after $2 \mathrm{~h}$, the respective phenyltelluro alcohol $\mathbf{4} \mathbf{j}$ and 
phenylseleno alcohol 4k in 77 and $86 \%$ yields respectively (Scheme 4). An excellent result was obtained with 2-(phenoxymethyl)oxirane $\mathbf{2 f}$, which afforded the respective phenyltelluteluro and phenylseleno alcohols in 82 and 93\% yields (Scheme 4, $\mathbf{4 h}$ and $\mathbf{4 i}$ ). In the reaction of propylene oxide $\mathbf{2 b}$ with dibutyl dichalcogenides $\mathbf{1 d}$ and $\mathbf{1 g}$, it was necessary 3.0 equiv of the dichalcogenide to obtain satisfactory yields of the respective alcohols in $2 \mathrm{~h}$ (Scheme $4, \mathbf{4 d}$ and $\mathbf{4 c}$ ).
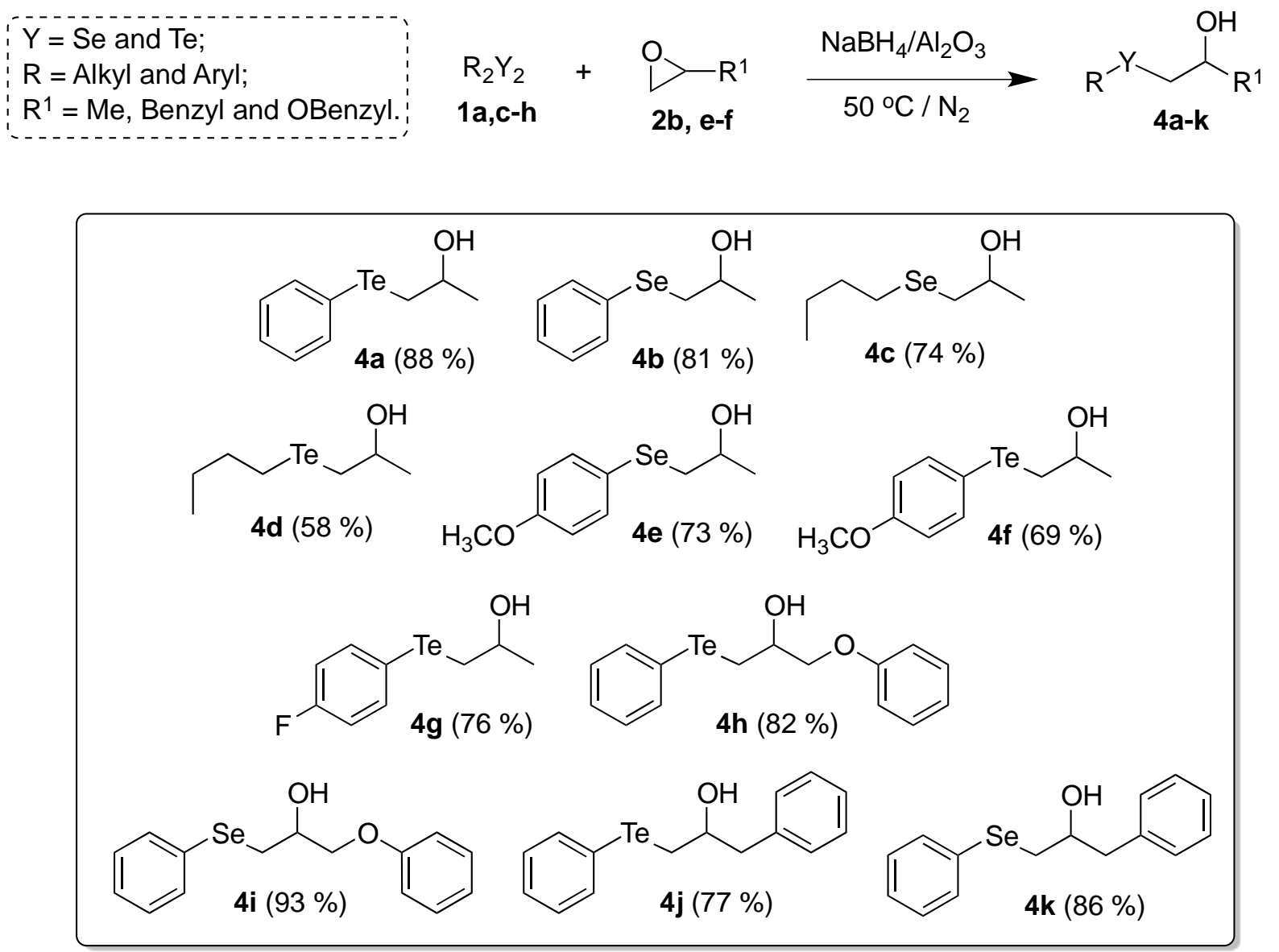

Scheme 4. Synthesis of chalcogen-containing alcohols $4 \mathbf{a}-\mathbf{k}$ by ring opening reactions of epoxides.

Next, we explored our protocol using $\mathrm{NaBH}_{4} / \mathrm{PEG}-400$ in the ring-opening of 2methylaziridine 2c, aiming to prepare $\beta$-chalcogen-containing amines $\mathbf{5 a - h}$ (Scheme 5). We found that the ring-opening of aziridine $\mathbf{2 c}$ is more efficient when nucleophilic selenium is used, producing the respective selenium-containing amines in better yields that the telluro-products. As in the ring-opening of lactone and epoxides (Schemes 2 and 4), the presence of electronwithdrawing or electron-releasing groups in the aromatic ring of the ditelluride and diselenide did not influence the yields of products in a predictable way. The yields of tellurium-containing aziridines ranged from 58 to $76 \%$, while the selenium-containing aziridines were obtained with yields from 69 to $85 \%$ (Scheme 5). Because of the basicity of the chalcogen-containing amines, in these reactions aqueous $\mathrm{NaCl}$ was used instead $\mathrm{NH}_{4} \mathrm{Cl}$ in the work up, to avoid product loss. 
Regarding the stability of the obtained chalcogen compounds, we have observed the following order: chalcogen-containing amines $>$ chalcogen-containing alcohols $>$ chalcogen-containing acids. In the presence of solvent, light or at high temperatures, the chalcogen-containing acids are decomposed. Thus, work-up and purification steps must be performed rapidly. The same care should be taken when working with the chalcogen-containing alcohols, but the degradation rate is lower. In contrast, the chalcogen-containing amines are very stable and do not require the same attention.

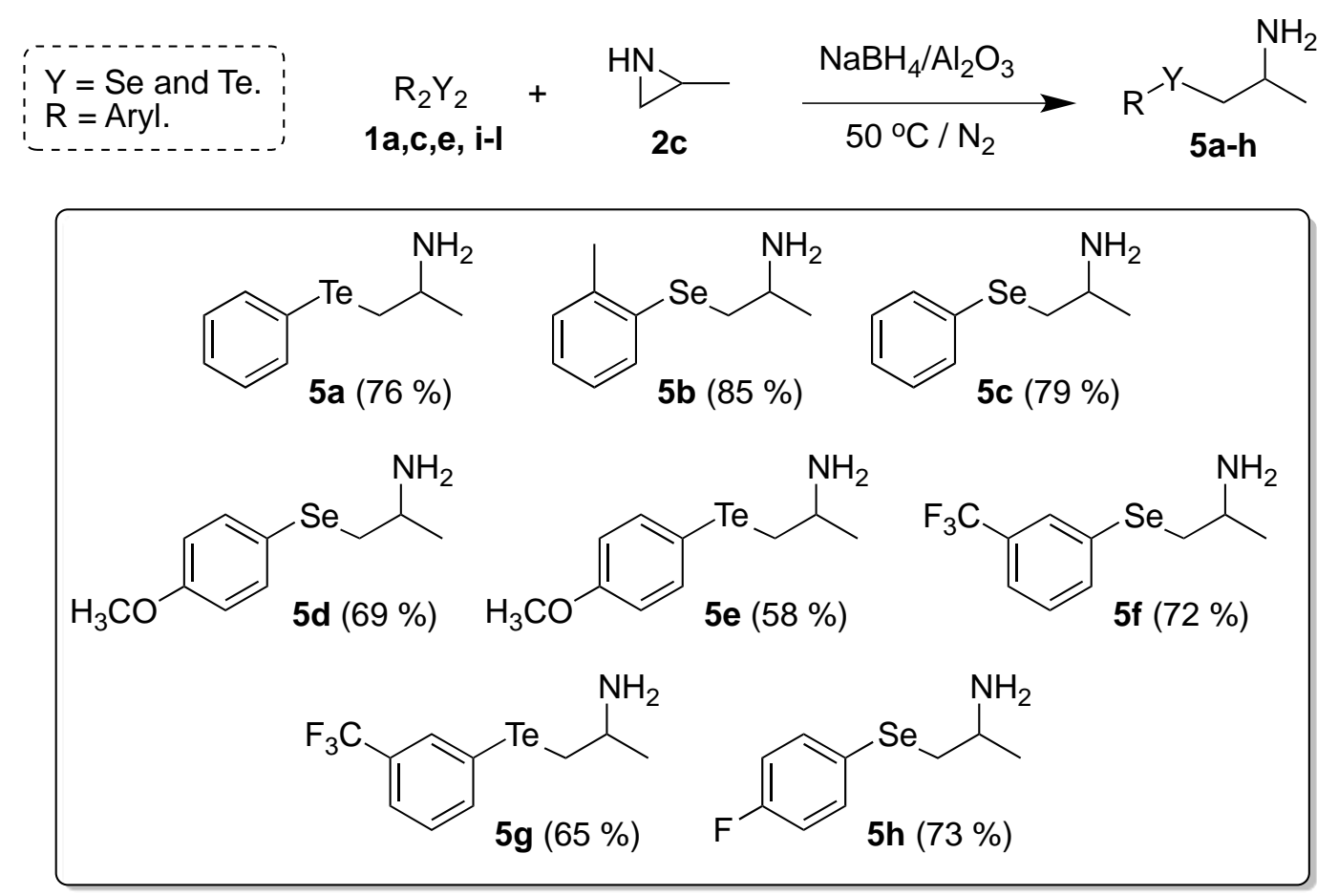

Scheme 5. Synthesis of chalcogen-containing amines $\mathbf{5 a - h}$ by ring opening reactions of 2methylaziridine.

\section{Conclusions}

In conclusion, we have shown that the use of $\mathrm{NaBH}_{4} / \mathrm{PEG}-400$ and $\mathrm{NaBH}_{4} / \mathrm{Al}_{2} \mathrm{O}_{3}$ as reducing systems to prepare chalcogenolate anions can be successfully applied in the synthesis of telluriumand selenium-functionalized acids, alcohols and amines. This atom-economic strategy involves the ring-opening of lactones, epoxides and aziridines and is general for dialkyl and diaryl ditellurides and diselenides. Moreover, this simple procedure does not involve harsh reaction conditions and is not time consuming, with good-to-excellent yields of products being obtained in only a twohour reaction. 


\section{Experimental Section}

Analytical thin-layer chromatography (TLC) was performed by using aluminum-backed silica plates coated with a $0.25 \mathrm{~mm}$ thickness of silica gel 60 F254 (Merck), visualized with an ultraviolet light $(\lambda=254 \mathrm{~nm})$. Either $300 \mathrm{MHz}$ or $500 \mathrm{MHz}$ acquired the NMR spectra. The ${ }^{1} \mathrm{H} \mathrm{NMR}$ chemical shifts are reported in parts per million (ppm) relative to tetramethylsilane (TMS) peak ( $\delta 0.0 \mathrm{ppm})$. The data are reported in chemical shift $(\delta)$, multiplicity, coupling constant $(J)$ in Hertz and integrated intensity. The ${ }^{13} \mathrm{C}$ NMR chemical shifts were reported at either 75 or $125 \mathrm{MHz}$ in ppm relative to $\mathrm{CDCl}_{3}$ signal $(\delta 77.0 \mathrm{ppm})$. The ${ }^{77} \mathrm{Se} \mathrm{NMR}$ chemical shifts are reported in ppm relative to internal standard $\mathrm{C}_{6} \mathrm{H}_{5} \mathrm{SeSeC}_{6} \mathrm{H}_{5}(\delta 467 \mathrm{ppm})$. The ${ }^{125} \mathrm{Te}$ NMR chemical shifts are reported in ppm relative to internal standard $\mathrm{C}_{6} \mathrm{H}_{5} \mathrm{TeTeC}_{6} \mathrm{H}_{5}(\delta 422 \mathrm{ppm})$. High-resolution mass spectra (HRMS) were acquired using a Bruker Daltonics MicroTOF instrument, operating electrospray ionization (ESI) mode with ion mass/charge $(\mathrm{m} / \mathrm{z}$ ) ratios as values in atomic mass units.

General procedure: To a $5 \mathrm{~mL}$ vial equipped with magnetic stirrer and a rubber septum under nitrogen, was added dialkyl or diaryl dichalcogenide $(0.5 \mathrm{mmol})$ and the electrophile $(1.5 \mathrm{mmol})$ followed by the catalyst system. To synthesize the chalcogen-containing acids and alcohols a $\mathrm{NaBH}_{4} / \mathrm{Al}_{2} \mathrm{O}_{3}(1 \mathrm{mmol} / 50 \mathrm{mg})$ system was employed and to prepare chalcogen-containing amines a NaBH $4 /$ PEG-400 ( $1 \mathrm{mmol} / 50 \mu \mathrm{L})$ system was used. The mixture was then stirred for $120 \mathrm{~min}$ at $50{ }^{\circ} \mathrm{C}$. The reaction progress was monitored by thin layer chromatography (TLC) and gas chromatography (GC). After $1 \mathrm{~h}$ at $\mathrm{rt}$ the reaction medium was diluted with AcOEt $(20 \mathrm{~mL})$ and washed with saturated aq solution of $\mathrm{NH}_{4} \mathrm{Cl}(15 \mathrm{~mL})$ for acids and alcohols and $\mathrm{NaCl}(15 \mathrm{~mL})$ for amines. The phases were separated and the aq phase was extracted with AcOEt $(2 \times 20 \mathrm{~mL})$. The organic phase was dried over $\mathrm{MgSO}_{4}$ and the solvents were evaporated under reduced pressure. The product was purified by flash column chromatography eluting first with hexane to remove alkyl or aryl chalcogen byproducts and then with hexane/AcOEt (8:2) to remove the acids or alcohols and AcOEt only to remove the amine.

3-(phenyltellanyl)butanoic acid (3a). Red oil, 83\% yield. ${ }^{1} \mathrm{H} \mathrm{NMR}\left(500 \mathrm{MHz}, \mathrm{CDCl}_{3}, 25{ }^{\circ} \mathrm{C}\right.$, TMS, $\delta \mathrm{ppm}$ ): 7.82-7.84 (m, 2H); 7.32-7.36 (m, 1H); 7.22-7.26 (m, 2H); 3.65 (sex, J 7.1 Hz, 1H); $2.86(\mathrm{dd}, J 6.7 \mathrm{~Hz}$ and $16.4 \mathrm{~Hz}, 1 \mathrm{H}) ; 2.82(\mathrm{dd}, J 7.7 \mathrm{~Hz}$ and $16.4 \mathrm{~Hz}, 1 \mathrm{H}) ; 1.64(\mathrm{~d}, J 7.15 \mathrm{~Hz}, 3 \mathrm{H})$. ${ }^{13} \mathrm{C}$ NMR (75 MHz, $\mathrm{CDCl}_{3}, 25{ }^{\circ} \mathrm{C}$, TMS, $\delta \mathrm{ppm}$ ): 178.0, 140.8, 129.2, 128.4, 111.2, 44.5, 24.2, 14.2. ${ }^{125} \mathrm{Te} \mathrm{NMR}\left(94.74 \mathrm{MHz}, \mathrm{CDCl}_{3}, 25{ }^{\circ} \mathrm{C}, \mathrm{C}_{6} \mathrm{H}_{5} \mathrm{TeTeH}_{5} \mathrm{C}_{6}\right.$ standard $\delta \mathrm{ppm} \mathrm{422):} \mathrm{709.4.} \mathrm{IR} v$ $\left(\mathrm{cm}^{-1}\right):$ 3064, 1708, 1573, 1433, 1297, 1222, 734, 693, 455. HR-MS: Calculated value $[\mathrm{M}+1]^{+}$ 294.9899; Found value $[\mathrm{M}+\mathrm{H}]^{+} 294.9912$.

3-(m-tolyltellanyl)butanoic acid (3b). Red oil, 92\% yield. ${ }^{1} \mathrm{H}$ NMR (300 $\mathrm{MHz}, \mathrm{CDCl}_{3}, 25{ }^{\circ} \mathrm{C}$, TMS, $\delta \mathrm{ppm}$ ): 7.81 (d, $J 7.4 \mathrm{~Hz}, 1 \mathrm{H}) ; 7.20-7.29(\mathrm{~m}, 2 \mathrm{H}) ; 7.02(\mathrm{dt}, J 1.4 \mathrm{~Hz}$, and $7.4 \mathrm{~Hz}, 1 \mathrm{H}) ; 3.70$ (sex, J 7.14 Hz, 1H); $2.85(\mathrm{~m}, 1 \mathrm{H}) ; 2.83(\mathrm{~d}, J 1.8 \mathrm{~Hz}, 1 \mathrm{H}) ; 2.51(\mathrm{~s}, 3 \mathrm{H}) ; 1.64(\mathrm{~d}, J 7.11 \mathrm{~Hz}, 3 \mathrm{H})$. ${ }^{13} \mathrm{C}$ NMR (75 MHz, $\mathrm{CDCl}_{3}, 25{ }^{\circ} \mathrm{C}$, TMS, $\left.\delta \mathrm{ppm}\right): 178.3,143.8,140.6,129.0,128.9,126.6,116.3$, 44.5, 27.6, 24.0, 14.0. IR $v\left(\mathrm{~cm}^{-1}\right): 3053,2862,2731,2627,1565,1341,1075,1049,989,931,909$, 
796, 705, 648, 603, 539, 477, 403. HR-MS: Calculated value $[\mathrm{M}+1]^{+}$309.0086; Found value $[\mathrm{M}$ $+\mathrm{H}]^{+} 309.011$.

3-((4-methoxyphenyl)tellanyl)butanoic acid (3c). Red crystals, $61 \%$ yield. ${ }^{1} \mathrm{H}$ NMR (300 MHz, $\mathrm{CDCl}_{3}, 25{ }^{\circ} \mathrm{C}$, TMS, $\left.\delta \mathrm{ppm}\right): 7.74(\mathrm{dd}, J 2.0 \mathrm{~Hz}$ and $6.6 \mathrm{~Hz}, 2 \mathrm{H}) ; 6.78(\mathrm{dd}, J 2.0 \mathrm{~Hz}$ and $6.6 \mathrm{~Hz}$, $2 \mathrm{H}) ; 3.80(\mathrm{~s}, 3 \mathrm{H}) ; 3.56(\mathrm{sex}, J 7.1 \mathrm{~Hz}, 1 \mathrm{H}) ; 2.80(\mathrm{~d}, J 0.6 \mathrm{~Hz}$ and $1.4 \mathrm{~Hz}, 2 \mathrm{H}) ; 1.59(\mathrm{~d}, J 7.1 \mathrm{~Hz}$, $3 \mathrm{H}) .{ }^{13} \mathrm{C}$ NMR (75 MHz, $\mathrm{CDCl}_{3}, 25{ }^{\circ} \mathrm{C}$, TMS, $\left.\delta \mathrm{ppm}\right): 178.1,160.2,143.0,115.1,100.3,55.1$, 44.5, 24.1, 13.9. IR $\left(\mathrm{cm}^{-1}\right): 3433,3016,2857,2732,2066,1967,1835,1563,1461,1397,1340$, 1133, 1102, 1064, 997, 911, 886, 789, 622, 588, 496, 418. HR-MS: Calculated value $[\mathrm{M}+1]^{+}$ 325.0005; Found value $[\mathrm{M}+\mathrm{H}]^{+} 324.9996$

3-(butyltellanyl)butanoic acid (3d). Red oil, 64\% yield. ${ }^{1} \mathrm{H} \mathrm{NMR}\left(500 \mathrm{MHz}, \mathrm{CDCl}_{3}, 25{ }^{\circ} \mathrm{C}, \mathrm{TMS}\right.$, $\delta \mathrm{ppm}): 3.48(\mathrm{sex}, J 7.1 \mathrm{~Hz}, 1 \mathrm{H}) ; 2.86(\mathrm{t}, J 6.6 \mathrm{~Hz}, 2 \mathrm{H}) ; 2.73(\mathrm{dt}, J 7.4$ and $3.1 \mathrm{~Hz}, 2 \mathrm{H}) ; 1.78$ (qt, $J 7.4 \mathrm{~Hz}, 2 \mathrm{H}) ; 1.68(\mathrm{~d}, J 7.2 \mathrm{~Hz}, 3 \mathrm{H}) ; 1.38(\mathrm{sex}, J 7.4 \mathrm{~Hz}, 2 \mathrm{H}) ; 0.92(\mathrm{t}, J 7.4 \mathrm{~Hz}, 3 \mathrm{H}) .{ }^{13} \mathrm{C}$ NMR (75 MHz, $\mathrm{CDCl}_{3}, 25{ }^{\circ} \mathrm{C}$, TMS, $\left.\delta \mathrm{ppm}\right): 178.3,45.4,34.4,25.3,24.9,13.4,8.5,3.5 . \mathrm{IR}\left(\mathrm{cm}^{-1}\right)$ : 2926, 2730, 1164, 1105, 1074, 990, 889, 769, 604, 496. HR-MS: Calculated value $[\mathrm{M}+1]^{+}$ 275.0212; Found value $[\mathrm{M}+\mathrm{H}]^{+} 275.0194$.

3-(phenylselanyl)butanoic acid (3e). Yellow oil, 85\% yield. ${ }^{1} \mathrm{H} \mathrm{NMR}\left(500 \mathrm{MHz}, \mathrm{CDCl}_{3}, 25{ }^{\circ} \mathrm{C}\right.$, TMS, $\delta$ ppm): 7.57-7.59 (m, 2H); 7.25-7.31 (m, 3H); $3.62(\mathrm{sex}, J 6.8 \mathrm{~Hz}, 1 \mathrm{H}) ; 2.73$ (dd, J 6.3 Hz and $16.1 \mathrm{~Hz}, 1 \mathrm{H}) ; 2.61(\mathrm{dd}, J 4.0 \mathrm{~Hz}$ and $16.1 \mathrm{~Hz}, 1 \mathrm{H}) ; 1.46(\mathrm{~d}, J 6.9 \mathrm{~Hz}, 3 \mathrm{H}) .{ }^{13} \mathrm{C} \mathrm{NMR}(75 \mathrm{MHz}$, $\mathrm{CDCl}_{3}, 25{ }^{\circ} \mathrm{C}$, TMS, $\left.\delta \mathrm{ppm}\right): 177.5,135.7,129.1,128.1,127.8,42.4,33.2,21.8$. IR $\left(\mathrm{cm}^{-1}\right): 3071$, 2731, 1952, 1880, 1605, 1595, 1499, 1377, 1110, 931, 813, 671, 471. HR-MS: Calculated value $[\mathrm{M}+1]^{+}$245.0002; Found value $[\mathrm{M}+\mathrm{H}]^{+} 244.9987$.

4-(phenylselanyl)butanoic acid (3f). Yellow oil, 79\% yield. ${ }^{1} \mathrm{H} \mathrm{NMR}\left(300 \mathrm{MHz}, \mathrm{CDCl}_{3}, 25{ }^{\circ} \mathrm{C}\right.$, TMS, $\delta$ ppm): 1.99 (quin, $(J 7.2 \mathrm{~Hz}, 2 \mathrm{H}), 2.51$ (t, J 7.2 Hz, 2H), 2.94 (t, J7.3 Hz, 2H), 7.24 (d, J8.5 $\mathrm{Hz}, 2 \mathrm{H}), 7.43$ (d, J 8.5 Hz , 2H), 11.50 (br s, 1H) ppm. ${ }^{13} \mathrm{C} \mathrm{NMR}\left(75 \mathrm{MHz}, \mathrm{CDCl}_{3}, 25{ }^{\circ} \mathrm{C}, \mathrm{TMS}\right.$, $\delta \mathrm{ppm}): 24.8,27.1,33.5,127.7,129.3,133.3,134.2,179.3 \mathrm{ppm}$.

1-(phenyltellanyl)propan-2-ol (4a). Red oil, 88\% yield. ${ }^{1} \mathrm{H} \mathrm{NMR}\left(500 \mathrm{MHz}, \mathrm{CDCl}_{3}, 25{ }^{\circ} \mathrm{C}, \mathrm{TMS}\right.$, $\delta \mathrm{ppm})$ : 7.73-7.75 (m, 2H), 7.25-7.29 (m, 1H), 7.18-7.21 (m, 2H), $3.91(\mathrm{sex}, J 5.8 \mathrm{~Hz}, 1 \mathrm{H}), 3.13$ $(\mathrm{dd}, J 4.5$ and $12.3 \mathrm{~Hz}, 1 \mathrm{H}), 2.96(\mathrm{dd}, J 7.6$ and $12.3 \mathrm{~Hz}, 1 \mathrm{H}), 1.29(\mathrm{~d}, J 6.1 \mathrm{~Hz}, 3 \mathrm{H}) .{ }^{13} \mathrm{C}$ NMR (125 MHz, $\mathrm{CDCl}_{3}, 25{ }^{\circ} \mathrm{C}$, TMS, $\delta \mathrm{ppm}$ ): 138.4, 129.3, 127.8, 111.1, 67.3, 23.7, 21.6. ${ }^{125} \mathrm{Te} \mathrm{NMR}$ (94.7 $\mathrm{MHz}, \mathrm{CDCl}_{3}, 25{ }^{\circ} \mathrm{C}, \mathrm{C}_{6} \mathrm{H}_{5} \mathrm{TeTeC}_{6} \mathrm{H}_{5}, \delta \mathrm{ppm}$ ): 365.8. HR-MS: Calculated value $[\mathrm{M}+23]^{+}$ 288.9950; Found value $[\mathrm{M}+\mathrm{Na}]^{+} 288.9937$.

1-(phenylselanyl)propan-2-ol (4b). Yellow oil, 81\% yield. ${ }^{1} \mathrm{H} \mathrm{NMR}\left(300 \mathrm{MHz}, \mathrm{CDCl}_{3}, 25{ }^{\circ} \mathrm{C}\right.$, TMS, $\delta \mathrm{ppm}$ ): 7.50-753 (m, 2H), 7.24-7.26 (m, 3H), 3.85 (sex, $J 3.48 \mathrm{~Hz}, 1 \mathrm{H}), 3.09$ (dd, $J 4.0$ and $12.7 \mathrm{~Hz}, 1 \mathrm{H}), 2.87(\mathrm{dd}, J 8.2$ and $12.7 \mathrm{~Hz}, 1 \mathrm{H}), 1.26(\mathrm{~d}, J 6.1 \mathrm{~Hz}, 3 \mathrm{H}) .{ }^{13} \mathrm{C} \mathrm{NMR}\left(75 \mathrm{MHz}, \mathrm{CDCl}_{3}\right.$, $25{ }^{\circ} \mathrm{C}$, TMS, $\left.\delta \mathrm{ppm}\right): 132.9,129.2,129.1,127.2,66.0,38.3,22.3 .{ }^{77} \mathrm{Se} \mathrm{NMR}\left(57 \mathrm{MHz}, \mathrm{CDCl}_{3}, 25\right.$ $\left.{ }^{\circ} \mathrm{C}, \mathrm{C}_{6} \mathrm{H}_{5} \mathrm{SeSeC}_{6} \mathrm{H}_{5}, \delta \mathrm{ppm}\right): 239.9 \mathrm{HR}-\mathrm{MS}$ : Calculated value $[\mathrm{M}+1]^{+}$217.0053; Found value [[M $+\mathrm{H}]^{+} 217.0062$.

1-(butylselanyl)propan-2-ol (4c). Yellow oil, $74 \%$ yield. ${ }^{1} \mathrm{H} \mathrm{NMR}\left(500 \mathrm{MHz}, \mathrm{CDCl}_{3}, 25{ }^{\circ} \mathrm{C}\right.$, TMS, $\delta \mathrm{ppm}$ ): 3.84 (sex, $J 6.1 \mathrm{~Hz}, 1 \mathrm{H}), 2.77$ (dd, $J 3.9$ and $12.7 \mathrm{~Hz}, 1 \mathrm{H}), 2.64$ (br s, 1H), 2.58 (dt, $J 3.4$ and $7.2 \mathrm{~Hz}, 2 \mathrm{H}), 2.53(\mathrm{dd}, J 8.4$ and $12.7 \mathrm{~Hz}, 1 \mathrm{H}), 1.64$ (qt, $J$ 7.2 Hz, 2H), 1.40 (sex, $J .45$ 
$\mathrm{Hz}, 2 \mathrm{H}), 1.27$ (d, J 6.2 Hz, 3H), 0.92 (t, $J 7.3 \mathrm{~Hz}, 3 \mathrm{H}) .{ }^{13} \mathrm{C} \mathrm{NMR}\left(125 \mathrm{MHz}, \mathrm{CDCl}_{3}, 25{ }^{\circ} \mathrm{C}, \mathrm{TMS}\right.$, $\delta \square \mathrm{ppm}): 65.9,34.6,32.7,24.2,22.9,22.4,13.5$. HR-MS: Calculated value $[\mathrm{M}+1]^{+}$197.0366; Found value $[\mathrm{M}+\mathrm{H}]^{+} 197.0372$.

1-(butyltellanyl)propan-2-ol (4d). Red oil, $58 \%$ yield. ${ }^{1} \mathrm{H} \mathrm{NMR}\left(500 \mathrm{MHz}, \mathrm{CDCl}_{3}, 25{ }^{\circ} \mathrm{C}, \mathrm{TMS}\right.$, $\delta \mathrm{ppm}$ ): 3.62 (sex, $J 6.0 \mathrm{~Hz}, 1 \mathrm{H}), 2.58(\mathrm{dd}, J 4.9$ and $12.0 \mathrm{~Hz}, 1 \mathrm{H}), 2.46(\mathrm{dd}, J 7.1$ and $12.0 \mathrm{~Hz}$, 1H), 2.37 (dt, $J 3.8$ and $6.8 \mathrm{~Hz}, 2 \mathrm{H}), 1.53(\mathrm{qt}, J 7.4 \mathrm{~Hz}, 2 \mathrm{H}), 1.20(\mathrm{sex}, J 7.4 \mathrm{~Hz}, 2 \mathrm{H}), 1.12(\mathrm{~d}, J$ $6.1 \mathrm{~Hz}, 3 \mathrm{H}), 0.79$ (t, J 7.35 Hz, 3H). ${ }^{13} \mathrm{C} \mathrm{NMR}\left(125 \mathrm{MHz}, \mathrm{CDCl}_{3}, 25{ }^{\circ} \mathrm{C}\right.$, TMS, $\delta \mathrm{ppm}$ ): 68.0, 35.0, 25.6, 24.3, 16.4, 13.9, 3.32. HR-MS: Calculated value $[\mathrm{M}+23]^{+}$269.0263; Found value $[\mathrm{M}+$ $\mathrm{Na}]^{+} 269.0262$.

1-((4-methoxyphenyl)selanyl)propan-2-ol (4e). Yellow oil, 73\% yield. ${ }^{1} \mathrm{H}$ NMR (300 MHz, $\mathrm{CDCl}_{3}, 25{ }^{\circ} \mathrm{C}$, TMS, $\left.\delta \mathrm{ppm}\right): 7.47(\mathrm{~d}, J 8.8 \mathrm{~Hz}, 2 \mathrm{H}), 6.81(\mathrm{~d}, J 8.7 \mathrm{~Hz}, 2 \mathrm{H}), 3.73-3.84(\mathrm{~m}, 1 \mathrm{H})$, $3.78(\mathrm{~s}, 3 \mathrm{H}), 2.99$ (dd, $J 3.9$ and $12.6 \mathrm{~Hz}, 1 \mathrm{H}), 2.77$ (dd, $J 8.4$ and $12.6 \mathrm{~Hz}, 1 \mathrm{H}), 2.59$ (br s, 1H), 1.24 (d, $J$ 6.1 Hz, 3H). ${ }^{13} \mathrm{C}$ NMR (75 MHz, $\mathrm{CDCl}_{3}, 25{ }^{\circ} \mathrm{C}$, TMS, $\delta$ ppm): 159.4, 135.8, 118.6, 114.8, 65.8, 55.2, 39.4, 22.2. ${ }^{77} \mathrm{Se} \mathrm{NMR}\left(57 \mathrm{MHz}, \mathrm{CDCl}_{3}, 25{ }^{\circ} \mathrm{C}, \mathrm{C}_{6} \mathrm{H}_{5} \mathrm{SeSeC}_{6} \mathrm{H}_{5}, \delta \mathrm{ppm}\right): 227.8$. CG-MS - $m / z^{+}$(relative intensity): 246 (88); 229 (30); 186 (100); 107 (28); 59 (17). HR-MS: Calculated value $[\mathrm{M}+1]^{+} 247.0159$; Found value $[\mathrm{M}+\mathrm{H}]^{+} 247.0102$.

1-((4-methoxyphenyl)tellanyl)propan-2-ol (4f). Yellow oil, 69\% yield. ${ }^{1} \mathrm{H}$ NMR (300 MHz, $\mathrm{CDCl}_{3}, 25{ }^{\circ} \mathrm{C}$, TMS, $\left.\delta \mathrm{ppm}\right): 7.68(\mathrm{~d}, J 7.2 \mathrm{~Hz}, 2 \mathrm{H}), 6.75(\mathrm{~d}, J 7.4 \mathrm{~Hz}, 2 \mathrm{H}), 3.86-3.91(\mathrm{~m}, 1 \mathrm{H})$, 3.77 (s, 3H), 3.04 (dd, $J 4.5$ and $12.1 \mathrm{~Hz}, 1 \mathrm{H}), 2.87$ (dd, $J 7.6$ and $12.1 \mathrm{~Hz}, 1 \mathrm{H}), 2.39$ (br s, 1H), 1.27 (d, J 6.1 Hz, 3H). ${ }^{13} \mathrm{C} \mathrm{NMR}\left(75 \mathrm{MHz}, \mathrm{CDCl}_{3}, 25{ }^{\circ} \mathrm{C}\right.$, TMS, $\delta$ ppm): 159.7; 140.9; 115.2; 99.9; 67.2; 55.0; 23.5; 21.7. ${ }^{125} \mathrm{Te} \mathrm{NMR}\left(94.7 \mathrm{MHz}, \mathrm{CDCl}_{3}, 25^{\circ} \mathrm{C}, \mathrm{C}_{6} \mathrm{H}_{5} \mathrm{TeTeC}_{6} \mathrm{H}_{5}, \delta \mathrm{ppm}\right)$ : 350.3. CGMS - $m / z^{+}$(relative intensity): 296 (94); 237 (48); 108 (100); 78 (15); 59 (11). HR-MS: Calculated value $[\mathrm{M}+1]^{+}$297.0056; Found value $[\mathrm{M}+\mathrm{H}]^{+} 297.0089$.

1-((4-fluorophenyl)tellanyl)propan-2-ol (4g). Yellow oil, 76\% yield. ${ }^{1} \mathrm{H}$ NMR (300 MHz, $\mathrm{CDCl}_{3}, 25^{\circ} \mathrm{C}$, TMS, $\left.\delta \mathrm{ppm}\right): 7.23(\mathrm{dd}, J 4.3 \mathrm{~Hz}$ and $6.6 \mathrm{~Hz}, 2 \mathrm{H}), 6.90(\mathrm{t}, J 6.69 \mathrm{~Hz}, 2 \mathrm{H}), 3.88-3.93$ (m, 1H), 3.09 (dd, $J 4.6$ and $12.2 \mathrm{~Hz}, 1 \mathrm{H}), 2.93$ (dd, $J 7.5$ and 12.2 Hz, 1H), 2.31 (br s, 1H), 1.29 $(\mathrm{d}, J 6.1 \mathrm{~Hz}, 3 \mathrm{H}) .{ }^{13} \mathrm{C} \mathrm{NMR}\left(75 \mathrm{MHz}, \mathrm{CDCl}_{3}, 25{ }^{\circ} \mathrm{C}\right.$, TMS, $\left.\delta \mathrm{ppm}\right): 162\left(\mathrm{~d}, J_{\mathrm{C}-\mathrm{F}} 246.6 \mathrm{~Hz}\right) ; 140.8$ $\left(\mathrm{d}, J_{\mathrm{C}-\mathrm{F}} 7.5 \mathrm{~Hz}\right) ; 116.6\left(\mathrm{~d}, J_{\mathrm{C}-\mathrm{F}} 20.8 \mathrm{~Hz}\right) ; 104.6\left(\mathrm{~d}, J_{\mathrm{C}-\mathrm{F}} 3.7 \mathrm{~Hz}\right) ; 67.3 ; 23.7 ; 21.9 .{ }^{125} \mathrm{Te} \mathrm{NMR}(94.7$ $\mathrm{MHz}, \mathrm{CDCl}_{3}, 25{ }^{\circ} \mathrm{C}, \mathrm{C}_{6} \mathrm{H}_{5} \mathrm{TeTeC}_{6} \mathrm{H}_{5}, \delta \mathrm{ppm}$ ): 369.6 (d, $J_{\mathrm{Te}-\mathrm{F}} 9.5 \mathrm{~Hz}$ ). CG-MS - $m / z^{+}$(relative intensity): 283 (100); 242 (55); 95 (53); 59 (28). HR-MS: Calculated value $[\mathrm{M}+1]^{+} 284.9856$; Found value $[\mathrm{M}+\mathrm{H}]^{+} 284.9832$.

1-phenoxy-3-(phenyltellanyl)propan-2-ol (4h). Yellow oil, 82\% yield. ${ }^{1} \mathrm{H}$ NMR (300 MHz, $\mathrm{CDCl}_{3}, 25{ }^{\circ} \mathrm{C}$, TMS, $\left.\delta \mathrm{ppm}\right): 7.72-7.75(\mathrm{~m}, 2 \mathrm{H}), 7.22-7.27(\mathrm{~m}, 3 \mathrm{H}) ; 7.14-7.19(\mathrm{~m}, 2 \mathrm{H})$; 6.91-6.96 $(\mathrm{m}, 1 \mathrm{H}) ; 6.82-6.85(\mathrm{~m}, 2 \mathrm{H}) ; 4.11-4.18(\mathrm{~m}, 1 \mathrm{H}) ; 4.02(\mathrm{dd}, J 7.2$ and $9.3 \mathrm{~Hz}, 1 \mathrm{H}) ; 3.96(\mathrm{dd}, J 9.0$ and $9.3 \mathrm{~Hz}, 1 \mathrm{H}) ; 3.16(\mathrm{~d}, J 6.2 \mathrm{~Hz}, 2 \mathrm{H}) ; 2.78$ (br d, $J 4.8 \mathrm{~Hz}, 1 \mathrm{H}) .{ }^{13} \mathrm{C} \mathrm{NMR}\left(75 \mathrm{MHz}, \mathrm{CDCl}_{3}, 25\right.$ ${ }^{\circ} \mathrm{C}$, TMS, $\left.\delta \square \mathrm{ppm}\right): 158.2 ; 138.3 ; 129.4 ; 129.2 ; 127.8 ; 121.1 ; 114.4 ; 111.4 ; 71.4 ; 70.0 ; 13.6 .{ }^{125} \mathrm{Te}$ NMR (94.7 MHz, $\mathrm{CDCl}_{3}, 25{ }^{\circ} \mathrm{C}, \mathrm{C}_{6} \mathrm{H}_{5} \mathrm{TeTeC}_{6} \mathrm{H}_{5}, \delta \mathrm{ppm}$ ): 389.3. CG-MS - $m / z$ (relative intensity): 356 (100); 207 (46); 133 (71); 107 (48); 91 (25); 77 (63). HR-MS: Calculated value $[\mathrm{M}+1]^{+}$ 359.0213; Found value $[\mathrm{M}+\mathrm{H}]^{+} 359.0191$. 
1-phenoxy-3-(phenylselanyl)propan-2-ol (4i). Yellow oil, 93\% yield. ${ }^{1} \mathrm{H}$ NMR (300 MHz, $\mathrm{CDCl}_{3}, 25^{\circ} \mathrm{C}$, TMS, $\delta$ ppm): 7.51-7.55 (m, 2H); 7.21-7.28 (m, 5H); 6.91-6.97 (m, 1H); 6.83-6.86 $(\mathrm{m}, 2 \mathrm{H}) ; 4.05-4.14(\mathrm{~m}, 1 \mathrm{H}) ; 4.03(\mathrm{dd}, J 4.1$ and $9.3 \mathrm{~Hz}, 1 \mathrm{H}) ; 3.99(\mathrm{dd}, J 5.8$ and $9.3 \mathrm{~Hz}, 1 \mathrm{H}) ; 3.21$ (dd, $J 5.6$ and $12.8 \mathrm{~Hz}, 1 \mathrm{H}) ; 3.12$ (dd, $J 6.8$ and $12.8 \mathrm{~Hz}, 1 \mathrm{H}) ; 2.78$ (br d, $J 4.4 \mathrm{~Hz}, 1 \mathrm{H}) .{ }^{13} \mathrm{C}$ NMR (75 MHz, $\mathrm{CDCl}_{3}, 25{ }^{\circ} \mathrm{C}$, TMS, $\left.\delta \mathrm{ppm}\right): 158.2 ; 132.8 ; 129.4 ; 129.2 ; 129.1 ; 127.2 ; 121.1 ; 114.4$; 70.3; 69.0; 31.7. ${ }^{77} \mathrm{Se} \mathrm{NMR}\left(94.7 \mathrm{MHz}, \mathrm{CDCl}_{3}, 25{ }^{\circ} \mathrm{C}, \mathrm{C}_{6} \mathrm{H}_{5} \mathrm{SeSeC}_{6} \mathrm{H}_{5}, \delta\right.$ ppm): 242.5. CG-MS m/z (relative intensity): 307 (96); 215 (100); 183 (28); 134 (59); 91 (30); 77 (42). HR-MS: Calculated value $[\mathrm{M}+1]^{+} 309.0316$; Found value $[\mathrm{M}+\mathrm{H}]^{+} 309.0351$.

1-phenyl-3-(phenyltellanyl)propan-2-ol (4j). Yellow oil, 77\% yield. ${ }^{1} \mathrm{H}$ NMR $(300 \mathrm{MHz}$, $\mathrm{CDCl}_{3}, 25{ }^{\circ} \mathrm{C}$, TMS, $\left.\delta \mathrm{ppm}\right)$ : 7.65-7.68 (m, 2H); 7.11-7.27 (m, 8H); 3.92-3.97 (m, 1H); 3.08 (dd, $J 4.5$ and $12.1 \mathrm{~Hz}, 1 \mathrm{H}) ; 2.97(\mathrm{dd}, J 7.4$ and $12.1 \mathrm{~Hz}, 1 \mathrm{H}) ; 2.86(\mathrm{dd}, J 5.4$ and $13.5 \mathrm{~Hz}, 1 \mathrm{H}) ; 2.78$ (dd, $J 7.2$ and $13.5 \mathrm{~Hz}, 1 \mathrm{H}) ; 2.37$ (br d, $J 3.0 \mathrm{~Hz}, 1 \mathrm{H}) .{ }^{13} \mathrm{C} \mathrm{NMR}\left(75 \mathrm{MHz}, \mathrm{CDCl}_{3}, 25{ }^{\circ} \mathrm{C}\right.$, TMS, $\delta$ ppm): $138.1 ; 137.8 ; 129.2 ; 129.1 ; 128.4 ; 127.6 ; 126.4 ; 111.4 ; 72.2 ; 44.0 ; 18.4 .{ }^{125}$ Te NMR (94.7 $\mathrm{MHz}, \mathrm{CDCl}_{3}, 25{ }^{\circ} \mathrm{C}, \mathrm{C}_{6} \mathrm{H}_{5} \mathrm{TeTeC}_{6} \mathrm{H}_{5}, \delta \mathrm{ppm}$ ): 377.4. CG-MS - $m / z$ (relative intensity): 340 (55); 207 (43); 91 (100); 77 (25). HR-MS: Calculated value $[\mathrm{M}+\mathrm{H}]^{+}$343.0263; Found value $\left[\mathrm{M}^{+}+1\right]$ : 343.0211 .

1-phenyl-3-(phenylselanyl)propan-2-ol (4k). Yellow oil, 86\% yield. ${ }^{1} \mathrm{H}$ NMR (300 $\mathrm{MHz}$, $\mathrm{CDCl}_{3}, 25{ }^{\circ} \mathrm{C}$, TMS, $\left.\delta \mathrm{ppm}\right)$ : 7.42-7.46 (m, 2H); 7.12-7.27 (m, 8H); 3.86-3.94 (m, 1H); 3.07 (dd, $J 4.2$ and $12.6 \mathrm{~Hz}, 1 \mathrm{H}) ; 2.90(\mathrm{dd}, J 7.9$ and $12.6 \mathrm{~Hz}, 1 \mathrm{H}) ; 2.85(\mathrm{dd}, J 5.8$ and $7.7 \mathrm{~Hz}, 1 \mathrm{H}) ; 2.75$ (dd, $J 6.9$ and $7.7 \mathrm{~Hz}, 1 \mathrm{H}) ; 2.49$ (br s, $1 \mathrm{H}) .{ }^{13} \mathrm{C} \mathrm{NMR}\left(75 \mathrm{MHz}, \mathrm{CDCl}_{3}, 25{ }^{\circ} \mathrm{C}, \mathrm{TMS}, \delta\right.$ ppm): $137.7 ; 132.6 ; 129.4 ; 129.3 ; 129.0 ; 128.4 ; 127.0 ; 126.4 ; 71.0 ; 42.7 ; 35.6 .{ }^{77} \mathrm{Se} \mathrm{NMR}(94.7 \mathrm{MHz}$, $\left.\mathrm{CDCl}_{3}, 25^{\circ} \mathrm{C}, \mathrm{C}_{6} \mathrm{H}_{5} \mathrm{SeSeC}_{6} \mathrm{H}_{5}, \delta \mathrm{ppm}\right): 240.7 . \mathrm{CG}-\mathrm{MS}-\mathrm{m} / z$ (relative intensity): 291 (31); 200 (61); 183 (74); 157 (56); 115 (95); 91 (100); 77 (18). HR-MS: Calculated value $[\mathrm{M}+1]^{+}$293.0366; Found value $[\mathrm{M}+\mathrm{H}]^{+} 293.0315$.

1-(phenyltellanyl)propan-2-amine (5a). Red oil, 76\% yield. ${ }^{1} \mathrm{H} \mathrm{NMR}\left(500 \mathrm{MHz}, \mathrm{CDCl}_{3}, 25{ }^{\circ} \mathrm{C}\right.$, TMS, $\delta \square \mathrm{ppm}$ ): 7.74 (dd, $J 1.0$ and $11.4 \mathrm{~Hz}, 2 \mathrm{H}), 7.25-7.28(\mathrm{~m}, 1 \mathrm{H}), 7.19$ (t, $J 7.55 \mathrm{~Hz}, 2 \mathrm{H}), 3.21$ (sex, $J 6.3 \mathrm{~Hz}, 1 \mathrm{H}), 3.07(\mathrm{dd}, J 6.0$ and $12.1 \mathrm{~Hz}, 1 \mathrm{H}), 3.01(\mathrm{dd}, J 6.7$ and $12.1 \mathrm{~Hz}, 1 \mathrm{H}), 1.26(\mathrm{~d}, J$ $6.0 \mathrm{~Hz}, 3 \mathrm{H}) .{ }^{13} \mathrm{C} \mathrm{NMR}\left(125 \mathrm{MHz}, \mathrm{CDCl}_{3}, 25^{\circ} \mathrm{C}\right.$, TMS, $\left.\delta \mathrm{ppm}\right): 138.4,129.3,127.8,111.4,48.2$, 23.4, 19.6. IR $v\left(\mathrm{~cm}^{-1}\right): 3314 ; 3104 ; 2934 ; 2870 ; 1556 ; 1445 ; 1088 ; 698$. CG-MS - $m / z^{+}$(relative intensity \%): 265 (1); 222 (16); 57 (13); 44 (100). HR-MS: Calculated value $[\mathrm{M}+1]^{+} 266.0110$; Found value $[\mathrm{M}+\mathrm{H}]^{+} 266.0118$.

1-(o-tolylselanyl)propan-2-amine (5b). Yellow oil, 85\% yield. ${ }^{1} \mathrm{H}$ NMR (500 $\mathrm{MHz}, \mathrm{CDCl}_{3}, 25$ ${ }^{\circ} \mathrm{C}$, TMS, $\left.\delta \mathrm{ppm}\right): 7.43(\mathrm{~d}, J 7.5 \mathrm{~Hz}, 2 \mathrm{H}), 7.16(\mathrm{~d}, J 7.1 \mathrm{~Hz}, 1 \mathrm{H}), 7.14(\mathrm{t}, J 6.4 \mathrm{~Hz}, 1 \mathrm{H}), 7.09$ (t, $J$ $6.4 \mathrm{~Hz}, 1 \mathrm{H}), 3.09$ (sex, J 6.3 Hz, 1H), 2.99 (dd, $J 4.7$ and $12.1 \mathrm{~Hz}, 1 \mathrm{H}), 2.77$ (dd, $J 8.2$ and 12.1 $\mathrm{Hz}, 1 \mathrm{H}), 2.42$ (s, 1H), 1.18 (d, J $6.3 \mathrm{~Hz}, 3 \mathrm{H}) .{ }^{13} \mathrm{C} \mathrm{NMR}\left(125 \mathrm{MHz}, \mathrm{CDCl}_{3}, 25{ }^{\circ} \mathrm{C}, \mathrm{TMS}, \delta \mathrm{ppm}\right)$ : 139.3, 131.7, 131.0, 129.9, 126.7, 126.4, 46.9, 37.9, 23.5, 22.4. IR (KBr) $v\left(\mathrm{~cm}^{-1}\right): 3351 ; 3058$; 2964; 2868; 1590; 1466; 1036; 749. CG-MS - m/z (relative intensity \%): 229 (3); 186 (18); 91 (20); 44 (100). HR-MS: Calculated value $[\mathrm{M}+1]^{+} 230.0369$; Found value $[\mathrm{M}+\mathrm{H}]^{+} 230.0358$.

1-(phenylselanyl)propan-2-amine (5c). Yellow oil, 79\% yield. ${ }^{1} \mathrm{H}$ NMR (500 $\mathrm{MHz}, \mathrm{CDCl}_{3}, 25$ ${ }^{\circ} \mathrm{C}$, TMS, $\left.\delta \mathrm{ppm}\right)$ : 7.51-752 (m, 2H), 7.23-7.26 (m, 3H), 3.09 (sex, J 4.9 Hz, 1H), 3.03 (dd, J 4.9 
and $12.2 \mathrm{~Hz}, 1 \mathrm{H}), 2.81(\mathrm{dd}, J 7.8$ and $12.2 \mathrm{~Hz}, 1 \mathrm{H}), 1.18(\mathrm{~d}, J 6.3 \mathrm{~Hz}, 3 \mathrm{H}) .{ }^{13} \mathrm{C}$ NMR $(125 \mathrm{MHz}$, $\mathrm{CDCl}_{3}, 25^{\circ} \mathrm{C}$, TMS, $\left.\delta \mathrm{ppm}\right): 132.7,129.9,129.0,126.9,46.6,38.8,23.2$. IR $(\mathrm{KBr}) v\left(\mathrm{~cm}^{-1}\right): 3367$; 3049; 2941; 2870; 1604; 1420; 1012; 776. CG-MS - $m / z^{+}$(relative intensity \%): 215 (2); 172 (22); 57 (9); 44 (100). HR-MS: Calculated value $[\mathrm{M}+1]^{+}$216.0213; Found value $[\mathrm{M}+\mathrm{H}]^{+} 216.0221$. 1-((4-methoxyphenyl)selanyl)propan-2-amine (5d). Yellow oil, 69\% yield. ${ }^{1} \mathrm{H} \mathrm{NMR} \mathrm{(300} \mathrm{MHz,}$ $\left.\mathrm{CDCl}_{3}, 25^{\circ} \mathrm{C}, \mathrm{TMS}, \delta \mathrm{ppm}\right): 7.48(\mathrm{~d}, J 8.7 \mathrm{~Hz}, 2 \mathrm{H}), 6.81$ (d, $\left.J 8.8 \mathrm{~Hz}, 2 \mathrm{H}\right), 3.78(\mathrm{~s}, 3 \mathrm{H}), 3.02$ (sex, $J 4.6 \mathrm{~Hz}, 1 \mathrm{H}) 2.93(\mathrm{dd}, J 4.5$ and $12.2 \mathrm{~Hz}, 1 \mathrm{H}), 2.69(\mathrm{dd}, J 8.8$ and $12.2 \mathrm{~Hz}, 1 \mathrm{H}), 1.14(\mathrm{~d}, J 6.27$ $\mathrm{Hz}, 3 \mathrm{H}) .{ }^{13} \mathrm{C} \mathrm{NMR}\left(75 \mathrm{MHz}, \mathrm{CDCl}_{3}, 25{ }^{\circ} \mathrm{C}\right.$, TMS, $\left.\delta \mathrm{ppm}\right): 159.3,135.6,119.7,114.7,55.2,46.5$, 40.1, 23.2. IR (KBr) $v\left(\mathrm{~cm}^{-1}\right): 3351 ; 3282 ; 2961 ; 2836 ; 1590 ; 1491 ; 1348 ; 1029 ; 825 ; 519$. CG-MS - $m / z^{+}$(relative intensity \%): 245 (12); 202 (32); 187 (17); 58 (17); 44 (100). HR-MS: Calculated value $[\mathrm{M}+1]^{+} 246.0318$; Found value $[\mathrm{M}+\mathrm{H}]^{+} 246.0284$.

1-((4-methoxyphenyl)tellanyl)propan-2-amine (5e). Yellow oil, 58\% yield. ${ }^{1} \mathrm{H} \mathrm{NMR}(500 \mathrm{MHz}$, $\mathrm{CDCl}_{3}, 25^{\circ} \mathrm{C}$, TMS, $\left.\delta \mathrm{ppm}\right): 7.68(\mathrm{~d}, J 8.7 \mathrm{~Hz}, 2 \mathrm{H}), 6.74(\mathrm{~d}, J 6.7 \mathrm{~Hz}, 2 \mathrm{H}), 3.77$ (s, 3H), 3.08 (sex, $J 6.2 \mathrm{~Hz}, 1 \mathrm{H}) 2.99(\mathrm{dd}, J 5.1$ and $11.9 \mathrm{~Hz}, 1 \mathrm{H}), 2.81(\mathrm{dd}, J 7.2$ and $11.90 \mathrm{~Hz}, 1 \mathrm{H}), 2.28(\mathrm{br} \mathrm{s}, 2 \mathrm{H})$, 1.17 (d, $J 6.3 \mathrm{~Hz}, 3 \mathrm{H}) .{ }^{13} \mathrm{C}$ NMR (125 MHz, $\mathrm{CDCl}_{3}, 25{ }^{\circ} \mathrm{C}$, TMS, $\left.\delta \mathrm{ppm}\right): 159.6,140.8,115.1$, 100.3, 55.0, 47.5, 24.2, 22.2. IR (KBr) $v\left(\mathrm{~cm}^{-1}\right): 3354 ; 3275 ; 2943 ; 2835 ; 1597 ; 1456 ; 1332 ; 1029$; 811; 533. CG-MS - $m / z^{+}$(relative intensity \%): 295 (5); 252 (44); 237 (11); 58 (10); 44 (100). HRMS: Calculated value $[\mathrm{M}+23]^{+} 318.0215$; Found value $[\mathrm{M}+\mathrm{Na}]^{+} 318.0180$.

1-((3-(trifluoromethyl)phenyl)selanyl)propan-2-amine (5f). Yellow oil, 72\% yield. ${ }^{1} \mathrm{H}$ NMR $\left(500 \mathrm{MHz}, \mathrm{CDCl}_{3}, 25^{\circ} \mathrm{C}\right.$, TMS, $\left.\delta \mathrm{ppm}\right): 7.75(\mathrm{~s}, 1 \mathrm{H}) ; 7.68(\mathrm{~d}, J 7.8 \mathrm{~Hz}, 1 \mathrm{H}), 7.47(\mathrm{~d}, J 7.8 \mathrm{~Hz}$, $1 \mathrm{H}), 7.36(\mathrm{t}, J 7.75 \mathrm{~Hz}, 1 \mathrm{H}), 3.13(\mathrm{sex}, J 4.9 \mathrm{~Hz}, 1 \mathrm{H}) 3.07$ (dd, $J 4.7$ and $12.1 \mathrm{~Hz}, 1 \mathrm{H}), 2.87$ (dd, $J$ 7.6 and $12.1 \mathrm{~Hz}, 1 \mathrm{H}), 1.56$ (br s, $2 \mathrm{H}), 1.18$ (d, $J 6.3 \mathrm{~Hz}, 3 \mathrm{H}) .{ }^{13} \mathrm{C} \mathrm{NMR}\left(125 \mathrm{MHz}, \mathrm{CDCl}_{3}, 25{ }^{\circ} \mathrm{C}\right.$, TMS, $\delta \mathrm{ppm}): 135.5\left(J_{\mathrm{C}-\mathrm{F} 3} 1.2 \mathrm{~Hz}\right), 131.5,131.2\left(J_{\mathrm{C}-\mathrm{F} 3} 32.5 \mathrm{~Hz}\right), 129.2,128.8\left(J_{\mathrm{C}-\mathrm{F} 3} 3.7 \mathrm{~Hz}\right), 123.6$ $\left(J_{\mathrm{C}-\mathrm{F} 3} 271.3 \mathrm{~Hz}\right), 123.5\left(J_{\mathrm{C}-\mathrm{F} 3} 3.7 \mathrm{~Hz}\right) . \mathrm{IR}(\mathrm{KBr}) v\left(\mathrm{~cm}^{-1}\right): 3352 ; 3284 ; 2966 ; 2872 ; 1579 ; 1328$; 1166; 959; 795; 695. CG-MS - m/z (relative intensity \%): 283 (1); 240 (20); 57 (8); 44 (100). HRMS: Calculated value $[\mathrm{M}+1]^{+} 284.0087$; Found value $[\mathrm{M}+\mathrm{H}]^{+} 284.0112$.

1-((3-(trifluoromethyl)phenyl)tellanyl)propan-2-amine (5g). Yellow oil, $65 \%$ yield. ${ }^{1} \mathrm{H}$ NMR $\left(500 \mathrm{MHz}, \mathrm{CDCl}_{3}, 25^{\circ} \mathrm{C}, \mathrm{TMS}, \delta \mathrm{ppm}\right): 7.96(\mathrm{~s}, 1 \mathrm{H}) ; 7.88(\mathrm{~d}, J 7.6 \mathrm{~Hz}, 1 \mathrm{H}), 7.4(\mathrm{~d}, J 7.8 \mathrm{~Hz}, 1 \mathrm{H})$, $7.29(\mathrm{t}, J 7.7 \mathrm{~Hz}, 1 \mathrm{H}), 3.12-3.18(\mathrm{~m}, 2 \mathrm{H}), 2.96(\mathrm{dd}, J 5.1$ and $13.3 \mathrm{~Hz}, 1 \mathrm{H}), 1.43$ (br s, $2 \mathrm{H}), 1.19$ $(\mathrm{d}, J 6.2 \mathrm{~Hz}, 3 \mathrm{H}) .{ }^{13} \mathrm{C} \mathrm{NMR}\left(125 \mathrm{MHz}, \mathrm{CDCl}_{3}, 25{ }^{\circ} \mathrm{C}, \mathrm{TMS}, \delta \mathrm{ppm}\right): 141.2,134.6\left(J_{\mathrm{C}-\mathrm{F} 3} 3.7 \mathrm{~Hz}\right)$, $131.0\left(J_{\mathrm{C}-\mathrm{F} 3} 31.2 \mathrm{~Hz}\right), 129.2,124.2\left(J_{\mathrm{C}-\mathrm{F} 3} 3.7 \mathrm{~Hz}\right), 123.5\left(J_{\mathrm{C}-\mathrm{F} 3} 271.2 \mathrm{~Hz}\right), 112.7$. IR $(\mathrm{KBr}) v\left(\mathrm{~cm}^{-1}\right)$ : $3351 ; 3279 ; 2953 ; 2881 ; 1591 ; 1302 ; 1196 ; 949 ; 778 ; 667$. CG-MS - $m / z^{+}$(relative intensity \%): 332 (1); 290 (25); 57 (16); 44 (100). HR-MS: Calculated value $[\mathrm{M}+23]^{+}$355.9984; Found value $[\mathrm{M}+\mathrm{Na}]^{+} 355.9923$.

1-((4-fluorophenyl)selanyl)propan-2-amine (5h). Yellow oil, 73\% yield. ${ }^{1} \mathrm{H}$ NMR (300 MHz, $\mathrm{CDCl}_{3}, 25{ }^{\circ} \mathrm{C}$, TMS, $\delta \mathrm{ppm}$ ): 7.50 (dd, $J 8.7$ and $\left.5.4 \mathrm{~Hz}, 2 \mathrm{H}\right) ; 6.96(\mathrm{t}, J 8.7 \mathrm{~Hz}, 2 \mathrm{H}$ ), 3.05 (sex, $J$ $6.3 \mathrm{~Hz}, 1 \mathrm{H}), 2.98(\mathrm{dd}, J 12.0$ and $4.6 \mathrm{~Hz}, 1 \mathrm{H}), 2.76(\mathrm{dd}, J 7.8$ and $12.0 \mathrm{~Hz}, 1 \mathrm{H}), 1.80$ (br s, 2H), $1.15(\mathrm{~d}, J 6.2 \mathrm{~Hz}, 3 \mathrm{H}) .{ }^{13} \mathrm{C} \mathrm{NMR}\left(75 \mathrm{MHz}, \mathrm{CDCl}_{3}, 25{ }^{\circ} \mathrm{C}, \mathrm{TMS}, \delta \mathrm{ppm}\right): 162.7\left(J_{\mathrm{C}-\mathrm{F}} 981.5 \mathrm{~Hz}\right)$, $135.2\left(J_{\mathrm{C}-\mathrm{F}} 31.5 \mathrm{~Hz}\right), 124.2\left(J_{\mathrm{C}-\mathrm{F}} 13.7 \mathrm{~Hz}\right), 116.1\left(J_{\mathrm{C}-\mathrm{F}} 85.3 \mathrm{~Hz}\right), 46.5,39.8,23.2$. IR $(\mathrm{KBr}) v\left(\mathrm{~cm}^{-}\right.$ $\left.{ }^{1}\right): 3351 ; 3281 ; 2964 ; 2870 ; 1584 ; 1487 ; 1226 ; 1157 ; 826 ; 591$. CG-MS - $m / z^{+}$(relative intensity 
\%): 233 (1); 190 (25); 109 (7); 44 (100). HR-MS: Calculated value $[\mathrm{M}+23]^{+}$256.0119; Found value $[\mathrm{M}+\mathrm{Na}]^{+} 256.0095$.

\section{Acknowledgements}

We thank the CNPq, CAPES, FAPERGS and FAPESP 2014/23362-8 project for financial support. We thank the Central Multiusuário - CEM/UFABC for NMR analyses. CNPq is also acknowledged for the fellowship for R.G.J., E.J.L. and G.P.

\section{References}

1. Devillanova, F. A. in Handbook of Chalcogen Chemistry, Ed. RSC Publishing, Cambridge, UK, 2007.

2. Beletskaya, I.; Moberg, C. Chem. Rev. 2006, 106, 2320-2354. https://doi.org/10.1021/cr050530j

3. Javier, L-F.; Marcos, F. P-B.; Antônio, A. S-P.; Augusto, C. G.; Bruno, A. S.; Princival, C.; Dos Santos, A. A. Dyes Pigments 2014, 110, 28-48. https://doi.org/10.1016/j.dyepig.2014.04.044

4. Wendler, E. P.; Dos Santos, A. A. Synlett 2009, 1034-1040.

5. Freudedahl, D. M.; Santoro, S.; Shahzad, S. A.; Santi, C.; Wirth, T. Angew. Chem. Int. Ed. 2009, 48, 8409-8411. https://doi.org/10.1002/anie.200903893

6. Cunha, R. L. O. R.; Gouvêa, I. E.; Feitosa, G. P. V.; Alves, M. F. M.; Brömme, D.; Comasseto, J. V.; Tersariol, I. L. S.; Juliano, L. Biol. Chem. 2009, 390, 1205-1212.

7. Perin, G.; Alves, D.; Jacob, R. G.; Barcellos, A. M.; Soares, L. K.; Lenardão, E. J. ChemistrySelect 2016, 2, 205-258. https://doi.org/10.1002/slct.201500031

8. Monti, B.; Santi, C.; Bagnoli, L.; Marini, F.; Sancineto, L. Curr. Green Chem. 2016, 3, 68-75. https://doi.org/10.2174/2213346103666160127003716

9. Gusarova, N. K.; Chernysheva, N. A.; Yas'ko, S. V.; Trofimov, B. A. J. Sulfur Chem. 2015, $36,526-534$. https://doi.org/10.1080/17415993.2015.1066375

10. Gusarova, N. K.; Chernysheva, N. A.; Yas'ko, S. V.; Klyba, L. V.; Trofimov, B. A. J. Sulfur Chem. 2016, 37, 488-500. https://doi.org/10.1080/17415993.2016.1191635

11. Chernysheva, N. A.; Yas'ko, S. V.; Gusarova, N. K.; Trofimov, B. A. Russian J. Org. Chem. 2016, 52, 1511-1513. https://doi.org/10.1134/S1070428016100237 
12. Santi, C.; Santoro, S.; Testaferri, L.; Tiecco, M. Synlett 2008, 1471-1474.

https://doi.org/10.1055/s-2008-1078408

13. Santi, C.; Santoro, S.; Battisteli, B.; Testaferri, L.; Tiecco, M. Eur. J. Org. Chem. 2008, 32, 5387-5390.

https://doi.org/10.1002/ejoc.200800869

14. Salman, S. M.; Schwab, R. S.; Alberto, E. E.; Vargas, J.; Dornelles, L.; Rodrigues, O. E. D.; Braga, A. L. Synlett 2011, 69-72.

15. Salman, S. M.; Narayanaperumal, S.; Schwab, R. S.; Bender, C. R.; Dornelles, L.; Rodrigues, O. E. D. RSC Adv. 2012, 2, 8478-8482. https://doi.org/10.1039/c2ra21488a

16. Nazari, M.; Movassagh, B. Tetrahedron Lett. 2009, 50, 438-441. https://doi.org/10.1016/j.tetlet.2008.11.036

17. Lenardão, E. J.; Silva, M. S.; Sachini, M.; Lara, R. G.; Jacob, R. G.; Perin, G. ARKIVOC 2009, $x i, 221-227$.

18. Perin, G.; Borges, E. L.; Alves, D. Tetrahedron Lett. 2012, 53, 2066-2069. https://doi.org/10.1016/j.tetlet.2012.02.028

19. Perin, G.; Borges, E. L.; Peglow, T. J.; Lenardão, E. J. Tetrahedron Lett. 2014, 55, 5652-5655. https://doi.org/10.1016/j.tetlet.2014.08.101

20. Perin, G.; Borges, E. L.; Rosa, P. C.; Carvalho, P. N.; Lenardão, E. J. Tetrahedron Lett. 2013, $54,1718-1721$. https://doi.org/10.1016/j.tetlet.2013.01.071

21. Vargas, F.; Comasseto, J. V. J. Organomet. Chem. 2009, 694, 122-126. https://doi.org/10.1016/j.jorganchem.2008.09.025

22. Silva, M. S.; Dos Santos, A. A.; Comasseto, J. V. Tetrahedron Lett. 2009, 50, 6498-6501. https://doi.org/10.1016/j.tetlet.2009.09.023 